\title{
Differentiating dark energy and modified gravity with galaxy redshift surveys
}

\author{
Yun Wang \\ Homer L. Dodge Department of Physics \& Astronomy, Univ. of Oklahoma, \\ 440 W. Brooks St., Norman, OK 73019, USA; wang@nhn.ou.edu
}

\begin{abstract}
The observed cosmic acceleration today could be due to an unknown energy component (dark energy), or a modification to general relativity (modified gravity). If dark energy models and modified gravity models are required to predict the same cosmic expansion history $H(z)$, they will predict different growth rate for cosmic large scale structure, $f_{g}(z)$. If gravity is not modified, the measured $H(z)$ leads to a unique prediction for $f_{g}(z), f_{g}^{H}(z)$, if dark energy and dark matter are separate. Comparing $f_{g}^{H}(z)$ with the measured $f_{g}(z)$ provides a transparent and straightforward test of gravity. We show that a simple $\chi^{2}$ test provides a general figure-of-merit for our ability to distinguish between dark energy and modified gravity given the measured $H(z)$ and $f_{g}(z)$. We find that a magnitude-limited NIR galaxy redshift survey covering $>10,000(\mathrm{deg})^{2}$ and the redshift range of $0.5<z<2$ can be used to measure $H(z)$ to $1-2 \%$ accuracy via baryon acoustic oscillation measurements, and $f_{g}(z)$ to the accuracy of a few percent via the measurement of redshift-space distortions and the bias factor which describes how light traces mass. We show that if the $H(z)$ data are fit by both a DGP gravity model and an equivalent dark energy model that predict the same $H(z)$, a survey area of $11,931(\mathrm{deg})^{2}$ is required to rule out the DGP gravity model at the 99.99\% confidence level. It is feasible for such a galaxy redshift survey to be carried out by the next generation space missions from NASA and ESA, and it will revolutionize our understanding of the universe by differentiating between dark energy and modified gravity.
\end{abstract}

\section{INTRODUCTION}

The observed cosmic acceleration today [1, 2] could be due to an unknown energy component (dark energy, e.g., [3]), or a modification to general relativity (modified gravity, e.g., [4, 5]). Ref. [6] contains reviews with more complete lists of references. Illuminating the nature of dark energy is one of the most exciting challenges in cosmology today.

The cosmic expansion history, $H(z)=(d \ln a / d t)(a$ is the cosmic scale factor), and the growth rate for cosmic large scale structure, $f_{g}(z)=d \ln \delta / d \ln a[\delta=$ $\left.\left(\rho_{m}-\overline{\rho_{m}}\right) / \overline{\rho_{m}}\right]$, are two functions of redshift $z$ that can be measured from cosmological data. They provide independent and complementary probes of the nature of the observed cosmic acceleration [7, 9]. The precisely measured $H(z)$ and $\Omega_{m}$ lead to a unique prediction for $f_{g}(z)$ in the absence of modified gravity, $f_{g}^{H}(z)$, if dark energy and dark matter are separate. Comparing $f_{g}^{H}(z)$ with the measured $f_{g}(z)^{\text {obs }}$ provides a transparent and straightforward test of gravity (see Fig.1). If gravity is not modified, $H(z)$ and $f_{g}(z)$ together provide stronger constraints on dark energy models [10].

Using the VVDS data, Ref. 11 demonstrated that a magnitude-limited galaxy redshift survey can be used to measure $f_{g}(z)$ via measurements of redshift-space distortion parameter

$$
\beta(z)=\frac{f_{g}(z)}{b(z)}
$$

and the bias parameter $b(z)$ (which describes how light traces mass) from galaxy clustering. In this paper we show that a feasible, sufficiently wide and deep magnitude-limited galaxy redshift survey will allow us

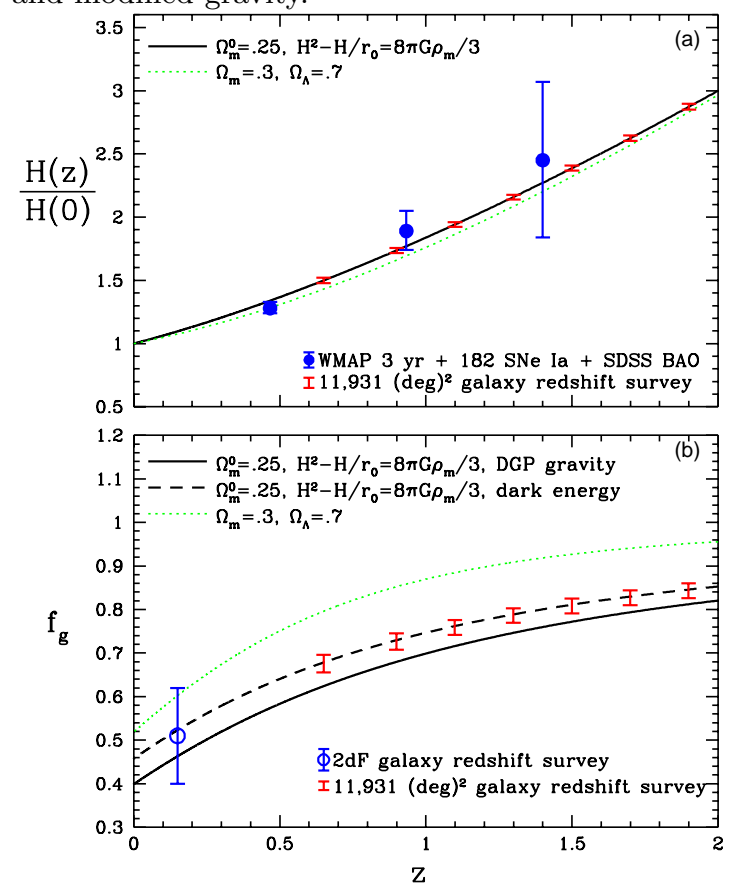

FIG. 1: Current and expected future measurements of the cosmic expansion history $H(z)=H_{0} E(z)$ and the growth rate of cosmic large scale structure $f_{g}(z)=d \ln \delta / d \ln a\left(\delta=\left(\rho_{m}-\overline{\rho_{m}}\right) / \overline{\rho_{m}}\right), a$ is the cosmic scale factor). Note that the fiducial model assumed for the future galaxy redshift survey is a dark energy model with the same $H(z)$ as that of the DGP model. These two models have identical expansion histories $H(z)$ [solid line in panel (a)], but very different growth rates $f_{g}(z)$ [solid and dashed lines in panel (b)].

to unambiguously differentiate between dark energy and modified gravity by providing precise measurements of $H(z)$ and $f_{g}(z)$ (see Fig.1). 


\section{MODELS}

If the present cosmic acceleration is caused by dark energy, $E(z) \equiv H(z) / H_{0}=\left[\Omega_{m}(1+z)^{3}+\Omega_{k}(1+z)^{2}+\right.$ $\left.\Omega_{X} X(z)\right]^{1 / 2}$, where $X(z) \equiv \rho_{X}(z) / \rho_{X}(0)$, with $\rho_{X}(z)$ denoting the dark energy density. The linear growth rate $f_{g} \equiv d \ln D_{1} / d \ln a$ is determined by solving the equation for $D_{1}=\delta^{(1)}(\mathbf{x}, t) / \delta(\mathbf{x})$,

$$
D_{1}^{\prime \prime}(\tau)+2 E(z) D_{1}^{\prime}(\tau)-\frac{3}{2} \Omega_{m}(1+z)^{3} D_{1}=0,
$$

where primes denote $d / d\left(H_{0} t\right)$, and we have assumed that dark energy and dark matter are separate.

In the simplest alternatives to dark energy, the present cosmic acceleration is caused by a modification to general relativity. The only rigorously worked example is the DGP gravity model [5, 7], which can be described by a modified Friedmann equation:

$$
H^{2}-\frac{H}{r_{0}}=\frac{8 \pi G \rho_{m}}{3}
$$

where $r_{0}$ is a parameter in DGP gravity, and $\rho_{m}(z)=$ $\rho_{m}(0)(1+z)^{3}$. Solving Eq.(3) gives

$E(z)=\frac{H(z)}{H_{0}}=\frac{1}{2}\left\{\frac{1}{H_{0} r_{0}}+\left[\frac{1}{\left(H_{0} r_{0}\right)^{2}}+4 \Omega_{m}^{0}(1+z)^{3}\right]^{1 / 2}\right\}$

with $\Omega_{m}^{0} \equiv \rho_{m}(0) / \rho_{c}^{0}, \rho_{c}^{0} \equiv 3 H_{0}^{2} /(8 \pi G)$. The added superscript " 0 " in $\Omega_{m}^{0}$ denotes that this is the matter fraction today in the DGP gravity model. Note that consistency at $z=0, E(0)=1$ requires that

$$
H_{0} r_{0}=\frac{1}{1-\Omega_{m}^{0}},
$$

so the DGP gravity model is parametrized by a single parameter, $\Omega_{m}^{0}$. The linear growth factor in the DGP gravity model is given by [7]

$D_{1}^{\prime \prime}(\tau)+2 E(z) D_{1}^{\prime}(\tau)-\frac{3}{2} \Omega_{m}(1+z)^{3} D_{1}\left(1+\frac{1}{3 \alpha_{D G P}}\right)=0$,

where

$$
\alpha_{D G P}=\frac{1-2 H_{0} r_{0}+2\left(H_{0} r_{0}\right)^{2}}{1-2 H_{0} r_{0}} .
$$

The dark energy model equivalent of the DGP gravity model is specified by requiring

$$
\frac{8 \pi G \rho_{d e}^{e f f}}{3}=\frac{H}{r_{0}} .
$$

Eq.(3) and the conservation of energy and momentum equation,

$$
\dot{\rho}_{d e}^{e f f}+3\left(\rho_{d e}^{e f f}+p_{d e}^{e f f}\right) H=0,
$$
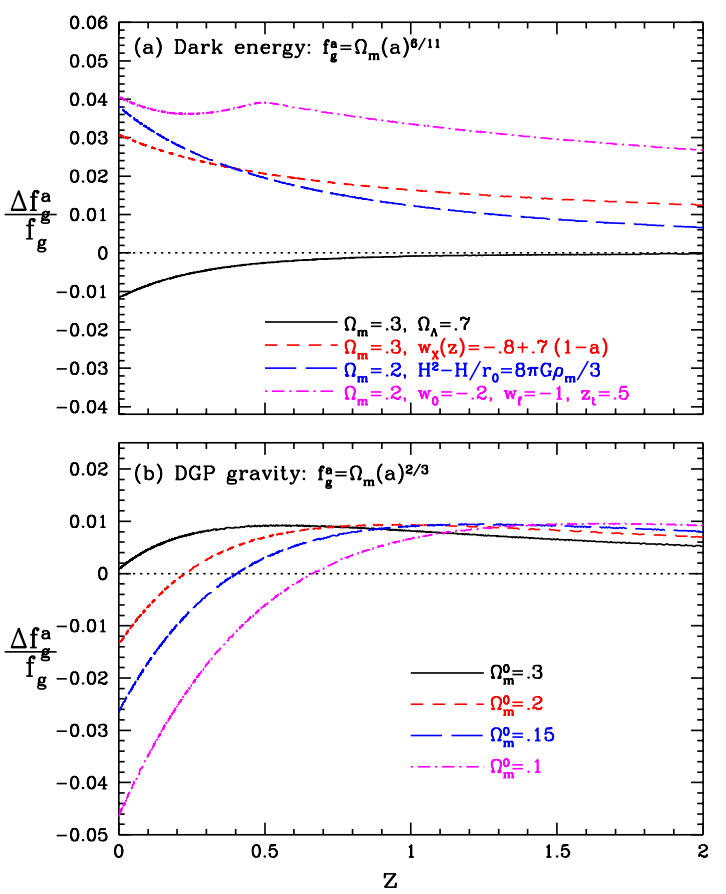

FIG. 2: The accuracy of approximate expressions for $f_{g}(z)$ for various models.

imply that [7]

$$
w_{d e}^{e f f}=-\frac{1}{1+\Omega_{m}(a)}
$$

where

$$
\Omega_{m}(a) \equiv \frac{8 \pi G \rho_{m}(z)}{3 H^{2}}=\frac{\Omega_{m}^{0}(1+z)^{3}}{E^{2}(z)}
$$

As $a \rightarrow 0, \Omega_{m}(a) \rightarrow 1$, and $w_{d e}^{e f f} \rightarrow-0.5$. As $a \rightarrow 1$, $\Omega_{m}(a) \rightarrow \Omega_{m}^{0}$, and $w_{d e}^{e f f} \rightarrow-1 /\left(1+\Omega_{m}^{0}\right)$. This means that the matter transfer function for the dark energy model equivalent of viable DGP gravity model $\left(\Omega_{m}^{0}<0.3\right.$ and $w \leq-0.5)$ is very close to that of the $\Lambda$ CDM model at $k \gtrsim 0.001 \mathrm{~h} \mathrm{Mpc}^{-1} \cdot[12]$

It is very easy and straightforward to integrate Eqs.(2) and (6) to obtain $f_{g}$ for dark energy models and DGP gravity models, with the initial condition that for $a \rightarrow$ $0, D_{1}(a)=a$ (which assumes that the dark energy or modified gravity are negligible at sufficiently early times). There are well known approximations to $f_{g}$, with $f_{g}(z)=\Omega_{m}(a)^{6 / 11}$ for dark energy models [13], and $f_{g}(z)=\Omega_{m}(a)^{2 / 3}$ for DGP gravity models 7]. Fig.2 shows that these powerlaw approximations of $f_{g}$ are not sufficiently accurate for future galaxy redshift surveys that can measure $f_{g}$ to a few percent accuracy in $\Delta z=0.2$ redshift bins. 


\section{ANALYSIS TECHNIQUE}

Galaxy redshift surveys allow us to measure both $H(z)$ and $f_{g}(z)$ through baryon acoustic oscillation (BAO) measurements [15, 16, 17, 32] and redshift-space distortion measurements [1]]. BAO in the observed galaxy power spectrum have the characteristic scale determined by the comoving sound horizon at recombination, which is precisely measured by the cosmic microwave background (CMB) anisotropy data [14]. Comparing the observed BAO scales with the expected values gives $H(z)$ in the radial direction, and $D_{A}(z)$ [the angular diameter distance $D_{A}(z)=r(z) /(1+z)$, where $r(z)$ is the coordinate or comoving distance] in the transverse direction. We will only estimate the accuracy to which $H(z)$ and $f_{g}(z)$ can be determined from galaxy redshift surveys in dark energy models (the error bars in Fig.1). ${ }^{1}$

The observed power spectrum is reconstructed using a particular reference cosmology, including the effects of bias and redshift-space distortions [16]:

$$
\begin{aligned}
P_{\text {obs }}\left(k_{\perp}^{r e f}, k_{\|}^{r e f}\right)= & \frac{\left[D_{A}(z)^{r e f}\right]^{2} H(z)}{\left[D_{A}(z)\right]^{2} H(z)^{r e f}} b^{2}\left(1+\beta \mu^{2}\right)^{2} \\
& \cdot\left[\frac{G(z)}{G(0)}\right]^{2} P_{\text {matter }}(k)_{z=0}+P_{\text {shot }},(12)
\end{aligned}
$$

where the growth factor $G(z)$ and the growth rate $f_{g}(z)=\beta b(z)$ are related via $f_{g}(z)=d \ln G(z) / d \ln a$, and $\mu=\mathbf{k} \cdot \hat{\mathbf{r}} / k$, with $\hat{\mathbf{r}}$ denoting the unit vector along the line of sight; $\mathbf{k}$ is the wavevector with $|\mathbf{k}|=k$. Hence $\mu^{2}=k_{\|}^{2} / k^{2}=k_{\|}^{2} /\left(k_{\perp}^{2}+k_{\|}^{2}\right)$. The values in the reference cosmology are denoted by the subscript "ref", while those in the true cosmology have no subscript. Note that

$$
k_{\perp}^{r e f}=k_{\perp} \frac{D_{A}(z)}{D_{A}(z)^{r e f}}, \quad k_{\|}^{r e f}=k_{\|} \frac{H(z)^{r e f}}{H(z)} .
$$

Eq.(12) characterizes the dependence of the observed galaxy power spectrum on $H(z)$ and $D_{A}(z)$ due to $\mathrm{BAO}$, as well as the sensitivity of a galaxy redshift survey to the redshift-space distortion parameter $\beta[21]$.

To study the expected impact of future galaxy redshift surveys, we use the Fisher matrix formalism. In the limit where the length scale corresponding to the survey volume is much larger than the scale of any features in $P(k)$, we can assume that the likelihood function for the band powers of a galaxy redshift survey is Gaussian 22]. Then the Fisher matrix can be approximated as 23]

$$
F_{i j}=\int_{k_{\min }}^{k_{\max }} \frac{\partial \ln P(\mathbf{k})}{\partial p_{i}} \frac{\partial \ln P(\mathbf{k})}{\partial p_{j}} V_{e f f}(\mathbf{k}) \frac{d \mathbf{k}^{3}}{2(2 \pi)^{3}}
$$

\footnotetext{
1 [18] gives a more precise treatment of redshift-space distortions, and [19] studies power spectra in alternative gravity models.
}

where $p_{i}$ are the parameters to be estimated from data, and the derivatives are evaluated at parameter values of the fiducial model. The effective volume of the survey

$$
\begin{aligned}
V_{\text {eff }}(k, \mu) & =\int d \mathbf{r}^{3}\left[\frac{n(\mathbf{r}) P(k, \mu)}{n(\mathbf{r}) P(k, \mu)+1}\right]^{2} \\
& =\left[\frac{n P(k, \mu)}{n P(k, \mu)+1}\right]^{2} V_{\text {survey }}
\end{aligned}
$$

where the comoving number density $n$ is assumed to only depend on the redshift for simplicity. Note that the Fisher matrix $F_{i j}$ is the inverse of the covariance matrix of the parameters $p_{i}$ if the $p_{i}$ are Gaussian distributed. Eq.(14) propagates the measurement error in $\ln P(\mathbf{k})$ (which is proportional to $\left[V_{e f f}(\mathbf{k})\right]^{-1 / 2}$ ) into measurement errors for the parameters $p_{i}$.

Since we do not include nonlinear effects, we only consider wavenumbers smaller than a minimum value of non-linearity. Following [15], we take $k_{\min }=0$, and $k_{\max }$ given by requiring that the variance of matter fluctuations in a sphere of radius $R, \sigma^{2}(R)=0.35$, for $R=\pi /\left(2 k_{\max }\right)$. We will also give results for $\sigma^{2}(R)=0.2$ for comparison. In addition, we impose a uniform upper limit of $k_{\max } \leq 0.2 \mathrm{hMpc}^{-1}$, to ensure that we are only considering the conservative linear regime essentially unaffected by nonlinear effects. 25] shows that nonlinear effects can be accurately taken into account. [26] shows that the BAO signal is boosted when these effects are properly included in the Hubble Volume simulation. We assume $\Omega_{b}=0.045, h=0.7, b=1$, and $n P=3[15]$; this is conservative since $n P>3$ at any redshift for a magnitude-limited survey.

The observed galaxy power spectrum in a given redshift shell centered at redshift $z_{i}$ can be described by a set of parameters, $\left\{H\left(z_{i}\right), D_{A}\left(z_{i}\right), \overline{G\left(z_{i}\right)}, \beta\left(z_{i}\right), P_{\text {shot }}^{i}, n_{S}\right.$, $\left.\omega_{m}, \omega_{b}\right\}$, where $n_{S}$ is the power-law index of the primordial matter power spectrum, $\omega_{m}=\Omega_{m} h^{2}$, and $\omega_{b}=\Omega_{b} h^{2}$ ( $h$ is the dimensionless Hubble constant). Note that $P(k)$ does not depend on $h$ if $k$ is in units of $\mathrm{Mpc}^{-1}$, since the matter transfer function $T(k)$ only depends on $\omega_{m}$ and $\omega_{b}$ [27], ${ }^{2}$ if the dark energy dependence of $T(k)$ can be neglected. Since $G(z), b$, and the power spectrum normalization $P_{0}$ are completely degenerate in Eq.(12), we have defined $\overline{G\left(z_{i}\right)} \equiv b G(z) P_{0}^{1 / 2} / G(0)$.

The square roots of diagonal elements of the inverse of the full Fisher matrix of Eq.(14) gives the estimated smallest possible measurement errors on the assumed parameters. The parameters of interest are $\left\{H\left(z_{i}\right), D_{A}\left(z_{i}\right)\right.$, $\left.\beta\left(z_{i}\right)\right\}$, all other parameters are marginalized over. Note that the estimated errors we obtain here are independent

\footnotetext{
2 Massive neutrinos can suppress the galaxy power spectrum amplitudes by $\gtrsim 4 \%$ on BAO scales [24]. It will be important for future work to quantify the effect of massive neutrinos on the measurement of $H(z)$ and $f_{g}(z)$.
} 
of cosmological priors, ${ }^{3}$ thus scale with (area) ${ }^{-1 / 2}$ for a fixed survey depth.

Fig. 1 shows the errors on $H(z)$ and $f_{g}(z)=\beta(z) b(z)$ for a dark energy model that gives the same $H(z)$ as a DGP gravity model with the same $\Omega_{m}^{0}$, for a redshift survey covering $11,931(\mathrm{deg})^{2}$, and the redshift range $0.5<z<2\left[\sigma^{2}(R)=0.35\right.$ assumed]. Note that the $D_{A}(z)$ measured from the same redshift survey provides additional constraints on $H(z)$ that can be used for crosschecking to eliminate systematic effects. We have neglected the very weak dependence of the transfer function on dark energy at very large scales in this model [12], and added $\Delta \ln b=0.01\left\{(\text { area }) /\left[28,600(\mathrm{deg})^{2}\right]\right\}^{-1 / 2}$ in quadrature to the estimated error on $\beta{ }^{4}$

[36] developed the method for measuring $b(z)$ from the galaxy bispectrum, which was applied by [37] to the $2 \mathrm{dF}$ data. Assuming that [35]

$$
\delta_{g}=b \delta(\mathbf{x})+\frac{1}{2} b_{2} \delta^{2}(\mathbf{x}),
$$

the galaxy bispectrum

$$
\begin{aligned}
\left\langle\delta_{g \mathbf{k}_{1}} \delta_{g \mathbf{k}_{2}} \delta_{g \mathbf{k}_{1}}\right\rangle= & (2 \pi)^{3}\left\{P _ { g } ( \mathbf { k } _ { 1 } ) P _ { g } ( \mathbf { k } _ { 2 } ) \left[\frac{J\left(\mathbf{k}_{1}, \mathbf{k}_{2}\right)}{b}\right.\right. \\
& \left.\left.+\frac{b_{2}}{b^{2}}\right]+c y c .\right\} \delta^{D}\left(\mathbf{k}_{1}+\mathbf{k}_{2}+\mathbf{k}_{3}\right),
\end{aligned}
$$

where $J$ is a function that depends on the shape of the triangle formed by $\left(\mathbf{k}_{1}, \mathbf{k}_{2}, \mathbf{k}_{3}\right)$ in $\mathbf{k}$ space, but only depends very weakly on cosmology [36].

Ref. 15] used Monte Carlo N-body simulation to study the extraction of the BAO scales. For comparison, we calculated $\left\{H\left(z_{i}\right), D_{A}\left(z_{i}\right)\right\}$ for the same fiducial model as considered by [15] (with the same assumptions and cutoffs in $k$ ), and obtained results that are within $30 \%$ of the values given by the fitting formulae from [28]. This is reassuring, as it validates the approach of using the Fisher matrix formalism to forecast the parameter accuracies for future redshift surveys. ${ }^{5}$

\section{OBSERVATIONAL METHODS}

$H(z)$ can be probed using multiple techniques. It can be measured using Type Ia supernovae (SNe Ia) as cosmological standard candles [29]. CMB and large scale

\footnotetext{
${ }^{3}$ Priors on $\omega_{m}, \omega_{b}, \Omega_{k}$, and $n_{S}$ will be required to obtain the errors on dark energy parameters.

4 This $\Delta \ln b$ estimate comes from extrapolating $2 \mathrm{dF}$ measurement of $b=1.04 \pm 0.11$ at $z \sim 0.15$ for an effective survey area of $1300 \times 127000 / 245591=672(\mathrm{deg})^{2}$ [37], and assuming a factor of 1.6 improvement for a NIR space mission that can detect galaxies at a much higher number density. This $\Delta \ln b$ estimate is comparable (and larger) than that estimated by [38] for imaging surveys at $z<2$.

${ }^{5}$ Ref. [20] found similar agreement in their comparison.
}

structure data provide constraints on cosmological parameters that help tighten the constraints on $H(z)$ [30]. Fig.1(a) shows the $H(z)$ given by Eq.(3) with $\Omega_{m}^{0}=0.25$ (solid line), as well as a cosmological constant model with $\Omega_{m}=0.3, \Omega_{\Lambda}=0.7$ (dotted line). Clearly, both these fit the constraints on $H(z)$ from current data [30] (no priors assumed) ${ }^{6}$

$\mathrm{BAO}$ measurements from a very wide and deep galaxy redshift survey provide a direct precise measurement of $H(z)$ [see Fig.1(a)]. Suppose $H(z)$ is measured to be $H^{2}-H / r_{0}=8 \pi G \rho_{m} / 3$ [see solid line in Fig.1(a)] and $\Omega_{m}$ is known accurately, Eq.(2) yields a unique prediction for $f_{g}(z), f_{g}^{H}(z)$, assuming that gravity is not modified [see the dashed line in Fig.1(b)].

The measurement of $f_{g}(z)$ can be obtained through independent measurements of $\beta=f_{g}(z) / b$ and $b(z)$ [11]. The parameter $\beta$ can be measured directly from galaxy redshift survey data by studying the observed redshiftspace correlation function [33, 34]. The bias factor $b(z)$ can be measured by studying galaxy clustering properties (for example, the galaxy bispectrum) from the galaxy redshift survey data [37]. Independent measurements of $\beta(z)$ and $b(z)$ have only been published for the $2 \mathrm{dF}$ data [33, 37, 39].

Fig.1(b) shows the $f_{g}(z)$ for the DGP gravity model with $\Omega_{m}^{0}=0.25$ (solid line), as well as a dark energy model that gives the same $H(z)$ for the same $\Omega_{m}^{0}$ (dashed line). The cosmological constant model from Fig.1(a) is also shown (dotted line). Clearly, current data can not differentiate between dark energy and modified gravity.

A very wide and deep galaxy redshift survey provides measurement of $f_{g}(z)$ accurate to a few percent [see Fig.1(b)]; this will allow an unambiguous distinction between dark energy models and modified gravity models that give identical $H(z)$ [see the solid and dashed lines in Fig.1(b)]. A simple $\chi^{2}$ test can provide a general figureof-merit for our ability to distinguish between dark energy and modified gravity models that fit the measured $H(z)$ but predict different $f_{g}(z)$. If the measurement errors are normally distributed, $\Delta \chi^{2} \equiv \chi^{2}(\mathbf{s})-\chi^{2}\left(\mathbf{s}_{0}\right)$ is distributed as a chi-square distribution with $n$ degrees of freedom ( $n$ is the number of data points), where $\mathbf{s}$ is the test model, and $\mathbf{s}_{0}$ is the bestfit model measured from data. $P\left(\chi^{2} \mid n\right)=99.99 \%$ corresponds to $\Delta \chi^{2}=29.877$ for $n=7$. Assuming that $\chi^{2}\left(\mathbf{s}_{0}\right)=n$, we find that $\chi^{2}(\mathbf{s})=36.877$. In Fig.1, we assume that the true model is a dark energy model with $\Omega_{m}^{0}=0.25$, $H^{2}-H / r_{0}=8 \pi G \rho_{m} / 3$, with $H r_{0}=1 /\left(1-\Omega_{m}^{0}\right)$. For a linear cutoff given by $\sigma^{2}(R)=0.35$ (or 0.2 ), a survey covering $11,931(\mathrm{deg})^{2}$ would rule out the DGP gravity model that gives the same $H(z)$ and $\Omega_{m}^{0}$ at $99.99 \%$ (or $95 \%)$ C.L.; a survey covering $13,912(\mathrm{deg})^{2}$ would rule out the DGP gravity model at $99.999 \%$ (or 99\%) C.L..

\footnotetext{
${ }^{6}$ Ref. [30] uses WMAP three year data [14], 182 type Ia supernovae [31], and the SDSS baryon acoustic scale measurement [32].
} 


\section{CONCLUSIONS}

Discovering the nature of dark energy has been identified as a high priority by both NASA and ESA. A magnitude-limited NIR galaxy redshift survey, covering $>10,000(\mathrm{deg})^{2}$ and the redshift range $0.5<z<2$, can be feasibly carried out by a space mission that uses MEMS technology to obtain 5000-10,000 galaxy spectra simultaneously [40, 41]. The low background from space enables very short exposure times to obtain galaxy spectra to $z \sim 2$, making it practical to carry out a magnitudelimited NIR galaxy redshift survey over $>10,000(\mathrm{deg})^{2}$ in only a few years. A magnitude-limited galaxy redshift survey over $>10,000(\mathrm{deg})^{2}$ will enable robust and precise determination of $b(z)$ using multiple techniques and with sufficient statistics [11, 36, 37]. This is critical for determining $f_{g}(z)$ using measurements of redshiftspace distortions. Such a survey will also enable rigorous study of the systematic uncertainties of BAO, and accurate measurements of redshift-space distortions.

Ref. [8] studied the use of weak lensing shear maps to differentiate between dark energy and modified gravity, complementary to what we have studied in this paper. While both weak lensing surveys and galaxy redshift surveys can provide accurate measurements of $H(z)$ (if the systematic uncertainties are properly modeled 7 Such a survey would allow us to distinguish between dark energy and modified gravity even if dark energy is clustered such that $f_{g}$, bias, and redshift distortions are scale-dependent [42], since a dark energy model and a modified gravity model generally have and controlled), galaxy redshift surveys can potentially provide the most accurate measurement of $f_{g}(z)$ [compare Fig.2 of [8] with Fig.1 of this paper, noting that $\left.f_{g}(z)=d \ln G(z) / d \ln a\right]$.

We have shown that a magnitude-limited NIR galaxy redshift survey covering $>10,000(\mathrm{deg})^{2}$ and $0.5<z<2$ can provide precise measurements of the cosmic expansion history $H(z)$, and the growth rate of cosmic large scale structure $f_{g}(z)$. These provide model-independent constraints on dark energy and the nature of gravity. The precisely measured $H(z)$ can be used to predict $f_{g}(z)$ expected in the absence of modified gravity, $f_{g}^{H}(z)$, if dark energy and dark matter are separate. Comparing $f_{g}^{H}(z)$ with $f_{g}(z)^{o b s}$ provides a transparent and powerful probe of modified gravity. This will allow us to illuminate the nature of the observed cosmic acceleration by differentiating between dark energy and modified gravity [see Fig.1]. A magnitude-limited survey covering $11,931(\mathrm{deg})^{2}$ can rule out the DGP gravity model at the $99.99 \%$ confidence level. ${ }^{7}$ If this technologically feasible survey is carried out by a space mission, it will have a revolutionary effect on our understanding of the universe.

Acknowledgements: I thank Gigi Guzzo for helpful comments on a draft of this paper, and Chris Blake, Craig Wheeler, and Eiichiro Komatsu for useful discussions.

different redshift dependences of the modified growth rate, and the data of such a survey can be analyzed in multi redshift slices, on multi scales, and using different populations of galaxies.
[1] A. G. Riess et al., Astron. J., 116, 1009 (1998)

[2] S. Perlmutter et al., ApJ, 517, 565 (1999)

[3] K. Freese et al., Nucl.Phys. B287, 797 (1987); P. J. E Peebles and B. Ratra, ApJ 325, L17 (1988); C. Wetterich, Nucl.Phys. B302, 668 (1988); J. A. Frieman et al., PRL 75, 2077 (1995); R. Caldwell, R. Dave, and P. J. Steinhardt, PRL 80, 1582 (1998);

[4] V. Sahni and S. Habib, PRL 81, 1766 (1998); L. Parker and A. Raval, PRD 60, 063512 (1999); C. Deffayet, Phys.Lett.B 502, 199 (2001); J-P. Uzan, F. Bernardeau, Phys. Rev. D64 (2001) 083004; K. Freese and M. Lewis, Phys.Lett.B 540, 1 (2002); V. K. Onemli and R. P. Woodard, PRD 70, 107301 (2004).

[5] G. Dvali, G. Gabadadze, M. Porrati, PLB, 485, 208 (2000)

[6] T. Padmanabhan, Phys.Rep. 380, 235 (2003); P. J. E Peebles and B. Ratra, Rev.Mod.Phys. 75, 55 (2003); V. Sahni and A. Starobinsky, IJMPD 15, 2105 (2006); E. J. Copeland, M. Sami, and S. Tsujikawa, IJMPD 15, 1753 (2006); P. Ruiz-Lapuente, Class. Quantum. Grav. 24, R91 (2007); B. Ratra and M. S. Vogeley, arXiv:0706.1565 (2007).V. Sahni, A. Starobinsky, IJMPD 15 (2006) 2105

[7] A. Lue, R. Scoccimarro, and G. D. Starkman, PRD 69, 124015 (2004); A. Lue, Physics Report 423, 1 (2006).

[8] L. Knox, Y. S. Song, , and J. A. Tyson, PRD, 74, 023512 (2006)

[9] A. F. Heavens, T.D. Kitching, L. Verde, MNRAS, 380,
1029 (2007); P. Zhang, M. Liguori, R. Bean, and S. Dodelson, Phys.Rev.Lett. 99 (2007) 141302; D. Sapone, L. Amendola, arXiv:0709.2792 [ps, pdf, other]

[10] R. A. Knop et al., ApJ 598, 102 (2003); Y. Wang and P. Mukherjee, ApJ 606, 654 (2004); Y. Wang and T. Tegmark, PRL 92, 241302 (2004).

[11] L. Guzzo, et al., Nature, 451, 541 (2008)

[12] C. P. Ma, R. R. Caldwell, P. Bode, and L. Wang, ApJ, 521, L1 ( 1999)

[13] L. Wang and P. J. Steinhardt, ApJ, 508, 483 (1998)

[14] D. N. Spergel et al., ApJS, 170, 377 (2007)

[15] C. Blake and K. Glazebrook, ApJ, 594, 665 (2003)

[16] H. Seo and D. J. Eisenstein, ApJ, 598, 720 (2003)

[17] See for example, M. White, Astropart. Phys. 24, 334 (2005); G. Huetsi, A\&A 449, 891 (2006); Y. Wang, ApJ 647, 1 (2006); R. Angulo et al., astro-ph/0702543 (2007).

[18] R. Scoccimarro, PRD, 70, 083007 (2004)

[19] H. F. Stabenau and B. Jain, PRD, 74, 084007 ( 2006)

[20] H. Seo and D. J. Eisenstein, ApJ, 633, 575 (2005)

[21] N. Kaiser, MNRAS, 227, 1 (1987)

[22] H. A. Feldman, N. Kaiser, and J. A. Peacock, ApJ, 426, 23 (1994)

[23] M. Tegmark, PRL, 79, 3806 (1997)

[24] D. J. Eisenstein and W. Hu, ApJ 511, 5 ( 1999); W. Hu, D. J. Eisenstein, and M. Tegmark, PRL 80, 5255 ( 1998).

[25] D. Jeong and E. Komatsu, ApJ 651, 619 ( 2006); M. Crocce, and R. Scoccimarro, arXiv:0704.2783; R. 
E. Smith, R. Scoccimarro, and R. K. Sheth, astro-ph/0703620

[26] R. S. Koehler, P. Schuecker, and K. Gebhardt, A\&A, 462, 7 (2007)

[27] D. Eisenstein and W. Hu, ApJ, 496, 605 (1998)

[28] C. Blake et al., MNRAS, 365, 255 (2006)

[29] M. M. Phillips, ApJ 413, L105 ( 1993);

[30] Y. Wang, and P. Mukherjee, Phys. Rev. D 76, 103533 (2007)

[31] P. Astier et al., Astron. Astrophys. 447, 31 (2006); A. G. Riess et al., ApJ 659, 98 (2007).

[32] D. Eisenstein et al., ApJ 633, 560 (2005);

[33] E. Hawkins et al., MNRAS, 346, 78 (2003)

[34] M. Tegmark et al., PRD 74, 123507 (2006); N. P. Ross et al., MNRAS 381, 573 (2007); J. da Angela et al., astro-ph/0612401.

[35] J. N. Fry and E. Gaztanaga, ApJ, 413, 447 ( 1993)
[36] S. Matarrese, L. Verde, and A. F. Heavens, MNRAS, 290, 651 ( 1997)

[37] L. Verde, et al., MNRAS, 335, 432 (2002); http://www.mso.anu.edu.au/2dFGRS/

[38] D. Dolney, B. Jain, and M. Takada, MNRAS, 366, 884 (2006)

[39] S. Nesseris, \& L. Perivolaropoulos, arXiv:0710.1092

[40] Y. Wang et al., BAAS v36, n5;1560 (2004); A. Crotts et al., astro-ph/0507043 (2005); E. Cheng and Y. Wang et al., Proc. of SPIE Vol. 6265, 626529 (2006).

[41] M. Robberto, A. Cimatti, and the SPACE science team, Venice 2007 Conf. Proc., to appear on Il Nuovo Cimento, arXiv:0710.3970

[42] W. Hu, PRD 65, 023003 (2002); C. Gordon, and W. $\mathrm{Hu}$, PRD 70, 083003 (2004); L. Hui, and K.P. Parfrey, arXiv:0712.1162 